Research Article,

\title{
Resonant Medicine
}

\author{
Praznikov Viktor MD, PhD \\ *Omer, Israel \\ Email Address: Praznikov@yandex.ru
}

\begin{abstract}
:
It was established for the first time that not only the resonance of destruction is presented in nature, but also the resonance of creation. In modern medicine, resonance and destruction resonance are widely represented. But only the first steps are being taken in describing and using the concept of "resonance of creation" for diagnostics and therapy (8-13). There is no description and physical interpretation of this concept. This review is an attempt to discuss the phenomenon of "resonance of creation" and the results of its use in the treatment of various diseases.
\end{abstract}

\section{Introduction:}

Resonance was discovered by Galeleo Galelei in 1604 (1). What is "resonance"? A platoon of soldiers approaches the wooden bridge and the officer gives the command to go out of step, because if a platoon of soldiers crosses the wooden bridge in step, the bridge can collapse from resonance. The vibrations of the bridge coincide with the vibrations of the marching soldiers, a resonance will arise, from which the bridge will collapse.

In this review, the role of the bridge is "played" by the disease, and the role of the marching soldiers is "performed" by the therapeutic effect. The soldier commander did not want the bridge to collapse due to the possible resonance. The physician, by contrast, absolutely needs resonance to destroy the disease.

From a technical point of view, resonance is a phenomenon of the response of an oscillatory system to an external influence. When the periods of influence and response of the system coincide, resonance occurs - a sharp increase in the amplitude of the oscillations under consideration.

Resonant methods for the study of matter have found wide application in physics, chemistry, biology, and medicine. For example, Nuclear Magnetic Resonance (NMR).

At the end of the 20th century, the method of magnetic resonance imaging (MRI) was developed on the basis of NMR. It is used to obtain images of the human brain, heart, and organs of the digestive tract. For the development of MRI in 2003, the American biophysicist Paul Lauterbur and his English colleague Peter Monsfield were awarded the Nobel Prize in Physiology or Medicine.

In addition to the resonance of destruction, the "resonance of communication, communication" is presented. Over the past decade at the University of California, Santa Barbara psychology professor Jonathan Schuler has developed what is called the "resonant theory of consciousness." Schooler, J.W., Smallwood, J., Christ off, K, Handy, T.C., Reichle, E.D., \& Sayette, M.A. (2011) Meta-awareness, perceptual decoupling and the wandering mind. Trends in Cognitive Sciences 15, 319-326 (2).

It is assumed that resonance - the term for synchronized vibrations - underlies not only human consciousness, but also the consciousness of animals and physical reality in general.

\section{Vibrations and everything connected with them.}

All things in our universe are constantly in motion, vibrating. Even objects that seem to be stationary actually vibrate, vibrate, resonate at different frequencies. When different vibrating things come together, 


\section{Praznikov Viktor / Resonant Medicine}

vibrations begin between the two states. And ultimately all matter is vibrations of various basic fields. Thus, on any scale, all nature is resonance, vibration.

Mathematician Stephen Strogatz cites various examples from physics, biology, chemistry, and neurobiology to illustrate this "synchronization" - his term for resonance - in his 2003 book "Synchronization: How Order Arises out of Chaos in the Universe, Nature, and Everyday Life":

* When some species of fireflies congregate in large clusters, they start blinking in sync, which can seem a little mysterious.

* When photons of the same power and frequency are synchronized, a laser beam is generated.

* The rotation of the Moon is precisely synchronized with its orbit around the Earth, so that we always see the same side.

The study of resonance leads to a potentially deep understanding of the nature of consciousness and the universe as a whole.

\section{Synchronization of vibrations in the head:}

Neuroscientists have also given their own definition of synchronization in their research. Large-scale firing of neurons occurs in the human brain at measurable frequencies, and it is believed that consciousness in mammals is usually associated with various types of neuronal synchronization.

For example, German neurophysiologist Pascal Fries investigated ways to synchronize different electrical patterns in the brain to study different types of human consciousness.

The main thesis is this: the special connections that provide large-scale consciousness are the result of a general resonance between many smaller components. The speed of the resonant waves present is the limiting factor that determines the size of each conscious entity at every moment.

But still, the principle of resonance in medicine is most often used today by homeopaths.

The principle of Homeopathy is based on the resonance between the homeopathic remedy and the disease. The name Homeopathy itself is derived from two words - "Homeo", i.e. similarly to "Pathos", i. e. disease. In other words, "like a disease." And what is "like a disease"? Like a disease is a medicine, a homeopathic remedy. The doctor finds the drug that in a healthy person, when taken, causes signs of the disease. And if so, then this medicine now in a sick person with such symptoms of the disease leads to a therapeutic result. The above is nothing more than a resonance. There is a resonance between the drug and the pathological process in a sick person (resonance pharmacology). As a result of resonance, the destruction of the disease occurs. At the same time, it must be recognized that homeopathy, which uses only low potencies of drugs, is not able to withstand extremely dangerous and incurable diseases - severe forms of oncology, diabetes mellitus, Parkinson's disease, Multiple sclerosis, etc.

In classical homeopathy, the physician evaluates the effectiveness of treatment with homeopathic remedies only by his own experience. Homeopaths use natural homeopathic medicines only in very low potencies (up to a potency of 1000).

This is due to the fact that high potency drugs lead to exacerbation of diseases. This circumstance extremely limits the possibility of their use, although there is an understanding that the higher the potency of the drug, the more effective it is.

So, the term "drug potency" is actively used not only in homeopathy but also in such a direction of medicine, which is called bioresonance therapy.

Let us briefly touch on what the "drug potency" is and how they are obtained. It has been established that the greater the potency of the drug, the higher its effectiveness.

Decimal dilutions were developed and introduced into homeopathic practice by the German physician Konstantin Goering (1800-1880). The centesimal dilutions were introduced by Samuel Hahnemann; the technology of their preparation was first described in detail in the 5th edition of the Organon (1833). LM (Q) potencies, dilution of 50,000, are also Hahnemann's inventions; they are described in the 6th edition of the Organon (1920). It is no coincidence that Hahnemann did not introduce large pyotencies of drugs - they caused an exacerbation of the course of diseases. 


\section{Praznikov Viktor / Resonant Medicine}

Without going into small details (you can learn about them from special reference books for the preparation of homeopathic remedies), the process of preparing liquid preparations can be briefly described as follows. A mother solution of the active substance is taken, part of which is mixed in a certain proportion with alcohol. If the ratio is one to ten, then the first decimal dilution is obtained, denoted in different countries D or X; if one in a hundred - the first centesimal, denoted by the letter $\mathrm{C}$ or not denoted at all.

For the preparation of subsequent dilutions, the corresponding part (tenth for decimal dilutions, hundredths for centesimal) of the resulting solution is taken, transferred to a new test tube and mixed again with the appropriate amount of alcohol, as described above for preparing the first dilutions

It has been shown that preparations of even greater dilution are effective on biological objects. So Professor Donders reports that one drop of atropine, brought to $1 / 700,000$, causes the pupil to dilate.

C. Darwin in his "Insectivorous Plants" gives reports on experiments on the action of weak solutions of phosphate ammonia on the plant Droserarotundifolia. It turned out that even one fourteen millionth part of a grain (a unit of measures of pharmaceutical weight equal to 0.0622 grams was used before the introduction of metric measures) (1/14 000000 , i.e. the amount corresponding to the seventh decimal dilution) still exhibits a very sharp effect on the vital activity of the leaves and tentacles of this plant.

Professor Arnold carried out experiments with strychnine, from which it can be seen that even one millionth part of a grain caused tetanus in frogs.

So, in addition to homeopathy, the principle of resonance in medicine is applied in a relatively new direction - bioresonance therapy. It consists of a diagnostic and a therapeutic part. The diagnostic part is called the Vegetative Resonance Test.

In 1975, the German physician Frank Morell came to a completely logical conclusion that if a disease of the organs of the human body is inevitably accompanied by disturbances in their frequency rhythm, then the essence of treatment should be to suppress the emerging "unhealthy" vibrations and restore normal ones.

The vegetative resonance test - ART, originally proposed in 1991 by the German scientist G. Schimmel (4), allows one point to be examined. Testing only one biologically active point with it makes it possible to assess the state of not only all organs and systems, but also their interconnection.

A computer-based device for bioresonance therapy was created, which included both diagnostic and therapeutic parts. The selector of a modern device for bioresonance therapy has a large selector with diagnostic (they are also therapeutic) markers, information copies of diseases, which are called "nosodes" when it comes to a disease, and "organ preparations" - information copies of healthy organs when a doctor deals with normal, non-pathological organs or parts thereof. "Nosodes" are necessary for the identification and treatment of diseases and "organ products" for testing perfectly healthy organs or parts of them. Nosodes are electronic markers about a disease and "organ products", information markers about a healthy organ or its part, recorded on a certain medium.

Each test drug has a wave effect on the patient. It is necessary to restore the spectral (frequency) harmony of the patient.

Original test preparations (as opposed to their information copies) are material objects, i.e. specific substances with an atomic-molecular structure characteristic of each of them. An inalienable property of matter is motion. Everything moves: from galaxies, stars and planets to the smallest particles of matter. In 1924, a French physicist, Louis de Broglie, expressed the hypothesis that was later confirmed that the waveparticle dualism established earlier for photons is inherent in all moving particles - electrons, protons, atoms, etc. the same as for photons $(5,6)$. Thus, moving particles of matter (substance) also have wave properties. So, the idea of waves associated not only with quanta of light, but also with massive particles was proposed by Louis de Broglie.

Louis de Broglie (1892-1987), was awarded the Nobel Prize (1929). in physics for the discovery of the wave nature of the electron. In 1923, developing A. Einstein's idea of a dual nature, he suggested that the flow of material particles should also have wave properties associated with their mass and energy (de Broglie waves). Experimental confirmation of this idea was obtained in 1927 in experiments on electron diffraction 
in crystals, and later it received practical application in the development of magnetic lenses for an electron microscope.

Waves associated with moving particles have nothing to do with the propagation of any electromagnetic field, i.e. to electromagnetic waves. In the physics of electromagnetic, acoustic and other waves, there is no analogue of waves associated with moving particles of matter. In other words, de Broglie waves are waves of a special nature. Particle wave theory has a lot to do with bioresonance therapy. All cells, organs and organ systems have wave characteristics, which is a manifestation of the material world, and they can be controlled by a device for bioresonance therapy.

So, bioresonance therapy is based on the wave theory of Louis de Broglie and is currently used in many countries. In bioresonance therapy, homeopathic medicines are used, as well as nosodes and organ preparations. All these remedies are presently present only in low potencies, just as in classical homeopathy.

\section{Resonance of destruction:}

\section{Diagnostics using fracture resonance}

In the practice of a doctor using bioresonance therapy, diagnostics are first performed. To do this, the nosode of the alleged disease is displayed on a computer screen connected to a device for bioresonance therapy, and it is tested in the patient. If the nosode is "not tested", then there is no resonance and the arrow on the computer screen does not fall down in the middle of the screen. Therefore, the patient does not have the disease that is displayed by the nosode. In the same case, if the nosode is tested, there is a resonance between the patient and the test drug - the arrow on the computer screen falls and indicates that the patient has the disease, the name of which is the nosode. This is a diagnostic resonance, but not a therapeutic one. This is how resonance diagnostics is carried out in bioresonance therapy.

\section{Healing using Destruction Resonance}

To treat the identified disease, the doctor must destroy either the tumor or the infectious process with the help of resonance, and for this it is necessary to potentiate the nosode identified in the patient, i.e. to find the potency of the nosode that will destroy the disease, in other words, a therapeutic resonance is needed. To do this, find that potency of the nosode (usually high), which leads to the fact that when testing this nosode in a patient, the arrow stops falling. Such a potency of the nosode leads to a resonant destruction of the structures of the disease. In other words, the informational content of the nosode in a certain potency is used for the resonant destruction of the structure of the disease, namely the treatment of the found disease. The doctor writes down the informational content of the potentiated nosode on the sugar crumbs and the patient takes this sugar crumbs and is thus treated, i.e. there is a resonant destruction of the structure of the disease.

The use of bioresonance therapy for the treatment of various diseases only of extremely low potencies, as in classical homeopathy, did not allow and does not allow to effectively treat many diseases, including oncological diseases, many infectious diseases, etc. In other words, for many years there has been a crisis in bioresonance therapy, and thus, in general, in resonance medicine. This can be seen in the materials of the annual scientific conferences on bioresonance therapy (7).

When it is said that drugs are used in works that exceed the LM potency of drugs, they mean those potencies that are prepared electronically (8-13).

The prepared classical homeopathic preparations and the electronic potentiation preparations, which are used in bioresonance therapy, do not fundamentally differ from each other. We have not seen in the literature indications of the difference between homeopathic preparations prepared by the usual, classical method and preparations of electronic potentiation.

Since 2016, materials have been published on the use of high potency drugs for treatment (8-13). It was found that drugs of high and ultra-high potency do not cause any side effects, including toxic effects on sick and healthy people. However, high potency drugs have proven to be extremely effective in the treatment of severe and extremely serious diseases such as cancer, infectious diseases, including HIV, stones and cysts in organs (8-13). In particular, metastatic forms of oncology are effectively treated. It has been established that all those forms of oncological diseases that are in the selector of the device for bioresonance therapy are effectively treated with drugs of high and ultra-high potencies. 


\section{Praznikov Viktor / Resonant Medicine}

Treatment of patients with nosode preparations exceeding the LM potency (hereinafter - in the F-potency) was not an end in itself. This method has been found in medical practice.

So, resonance medicine, in addition to homeopathy, includes resonance diagnostics and resonance therapy. Treatment of patients in whom the destruction of the structure of the disease, for example, oncology, occurs, is called the "resonance of destruction".

Destruction resonance effectively treats not only oncological diseases, but also prion diseases, fungal diseases, psoriasis, calculous process in the gallbladder and kidneys, brain and kidney cysts (8-13).

\section{Resonance of Creation}

Since 2016, materials have been published on the use of the second direction of therapeutic resonance "resonance of creation" (8-13). Resonance can not only destroy, for example, diseases, but also create, for example, create lost biological structures. This made it possible to treat degenerative diseases.

We could not find in the scientific literature an idea of that resonance can be not only a "resonance of destruction", but also a "resonance of creation." This is obviously due to the fact that it is not easy to imagine how the coincidence of frequencies leads to a response that is not destructive, but constructive. In this review, we have presented illustrations of how resonance can be not only destructive, but also constructive, in particular for the treatment of degenerative diseases.

In the treatment with the help of the resonance of destruction, nosodes of diseases were used, from which preparations in the F potency were prepared. This principle has not been effective in the treatment of degenerative diseases. The creation and formation of the principle of "resonance of creation" became possible only as a result of the fact that not nosodes were used for treatment, but oral preparations exceeding the LM potency. Without organ preparations in the F potency, it is impossible to imagine the use of this principle.

This review presents material related to the treatment of degenerative diseases. This means that treatment is nothing more than the process of restoring organs or organ systems that have undergone changes as a result of diseases or as a result of an aging degenerative process. Degenerative diseases can also be congenital. It is clear that a significant part of congenital diseases is a consequence of the underdevelopment of an organ or organ system. In practice, most often after an illness, for example, inflammation, or as a result of the senile process, the level of health of the organ falls down to its destruction. Such an organ requires restoration (rehabilitation). The resonance of creation makes it possible to restore an organ or part of it. Organ preparations are wave preparations (wave copies) of healthy organs or parts of them. Nosodes are wave drugs of the disease. In the selectors of hardware and software complexes for bioresonance therapy, there are various organ preparations. For the restoration and rehabilitation of organs, we used organ preparations in the F potency. They were done in the same way as the F potency nosodes.

\section{Diagnostics of the degenerative process by the method Vegetative resonance test}

When a patient is tested for a destroyed organ by a tumor, degenerative processes or inflammation, it is no longer tested as normal. In other words, it is tested as problematic. those. on the computer screen, an arrow falls in the middle of the screen, which indicates that the organopreparation has been found correctly. There is a resonance between the organopreparation and the patient. This is a diagnostic resonance, but not a therapeutic one.

\section{Treatment of the degenerative process using the resonance method Creation:}

We find the potency of the organopreparation that leads to resonance with the affected organ, namely, the termination of testing this organ or organ section as problematic. In this case, the arrow stops falling on the computer screen. This is a therapeutic resonance, but not diagnostic. The doctor prepares preparations of healthy organs in the F potency for the patient, writes them on the sugar crumbs, which the patient takes.

Parkinson's disease (PD). Clinical manifestations of PD are expressed in 1. tremor, 2. hypokinesia, 3. muscle rigidity, 4. postural instability. In the hyperkinetic form of PD, skeletal muscle tone decreases $(14,15)$. PD is a degenerative disease of the extrapyramidal motor system. The formations of the midbrain are affected - the red nucleus, the black matter, the reticular formation, the plate of the quadruple, the amygdala (limbic system). In the hyperkinetic form of PD, the striopalid system - the caudate and lenticular 


\section{Praznikov Viktor / Resonant Medicine}

nuclei - is involved in the pathological process. PD is a classic example of a degenerative disease. In the affected structures, the number of neurons sharply decreases, and, as a result, the dopamine content in them decreases. In allopathic medicine, the treatment of the disease is carried out according to the principle of substitution therapy. Patients are taking dopamine medications. It is clear that this principle of therapy cannot lead to a cure for PD. It is completely unproductive to use nosodes of this disease in the treatment of PD.

Since PD is a classic example of a degenerative disease, the principle of resonance of creation is applied to treat it. All subcortical formations that have undergone degeneration are shown for recovery. For this, organopreparations of these structures are used in the F potency.

In PD, those brain structures that are involved in the pathological process in Alzheimer's disease (AD) are also tested, namely: the amygdala, basal nucleus, islet cortex, cerebral septum, hippocampus, neocortex, temporal lobe of the brain, parietal lobe of the brain, isocortex, piriform share, insular field. At the same time, those features of the disease that are characteristic of $\mathrm{AD}$ (with early and moderate dementia) are revealed. It follows from the above that the treatment process is not limited to the restoration of the structures of the extrapyramidal system (PS), but also of all those structures that are tested and characteristic of AD. For treatment, organopreparations of these structures in the F potency are used.

As a result of treating patients with the method of creative resonance, degenerated structures are restored (they are no longer tested) and all the symptoms characteristic of Parkinson's disease are lost (10-13).

\section{Alzheimer disease}

Alzheimer's disease is a progressive form of senile dementia, leading to a complete loss of cognitive abilities, developing mainly after 60-65 years. Clinically manifests itself as a gradually and constantly progressive disorder of cognitive abilities: attention, memory, speech, praxis, gnosis, psychomotor coordination, orientation and thinking $(16,17)$.

In typical cases, Alzheimer's disease manifests itself in people over 60-65 years old. Alzheimer's type dementia is characterized by a subtle and prolonged onset, steady progression without periods of improvement. The main substrate of the disease is disorders of the higher nervous functions.

\section{Resonant diagnosis of Alzheimer's disease}

In the selector of the device, we find the following organ preparations of degenerated formations of the brain in $\mathrm{AD}$, necessary for treatment: amygdala, basal nucleus, insular cortex, cerebral septum, hippocampus, neocortex, temporal lobe, parietal lobe, isocortex, pear-shaped lobe, insular field, pre-base of the hippocampus, paralimbic area of the cortex. All of these structures are tested in patients, i.e. the arrow falls when testing the listed organopreparations in patients. The nosode is also tested - Amyloid or Amyloidosis, brain plaques (neurotic plaques), prions. We are testing atherosclerosis as an extremely important cause of $\mathrm{AD}$. In $\mathrm{AD}$, as a rule, those structures are tested that are also tested in Parkinson's disease - the black medulla, the reticular formation, the quadruple plate, etc., which are included in the treatment.

It is important to pay attention to the fact that the identification by testing of the above structures of the brain (they are tested) is necessary for the diagnosis of AD. There have been cases when the doctor does not detect any clinical symptoms of $\mathrm{AD}$, but tests the given configuration of the brain structures on the device. A similar situation is noted when a doctor tests a different configuration of brain structures, characteristic of Parkinson's disease, even in the period of the disease when there are no clinical manifestations of the disease. In this one can see very great possibilities of bioresonance diagnostics - vegetative resonance test (13).

\section{"Treatment of autoimmune disease by the resonance of creation".}

After testing, resonance diagnostics, all of the listed organopreparations, treatment is carried out by the method of resonance creation of $\mathrm{AD}$. The corresponding preparations are prepared from the tested organopreparations. They are recorded on sugar crumbs in the potency that is necessary for treatment and we carry out resonant treatment of patients.

Treatment of patients with early and moderate dementia. Treatment of all tested brain structures showed that the patient responds to this treatment quite adequately. In the course of treatment, the insular cortex was the first to be tested, followed by the nucleus basalis. As the testability of these brain structures decreased, the patient reported that his condition was getting significantly better, not only in terms of recovering short-term 
memory, but also in other indicators. In patients with moderate dementia, in the first weeks of treatment, organopreparations, the islet cortex, the basal nucleus, the temporal lobe, the isocortex, and those characteristic of Parkinson's disease - the lenticular nucleus, paranigral dopamine nuclei of the middle brain " "roof of the midbrain".

Subsequently, the hippocampus, piriform lobe, islet field, and hippocampal pre-basement were no longer tested. At the same time, the reports of the patients' relatives changed: "Our patient began to cry less, take offense at us less and began to recognize her close people more often - children, grandchildren. It is also very important that it became easier for her to get up from the chair. Before starting treatment, getting up from a chair was a difficult and time-consuming process for her. She became less resentful, and urinary incontinence became less common». Treatment is carried out until complete loss of AD symptoms.

The foregoing testifies to the effective treatment of AD by the method of resonance of creation (13).

Treatment of diseases with a combined method - the resonance of destruction and the resonance of creation.

\section{Treating autoimmune diseases resonance of creation}

Autoimmune diseases are a wide class of diseases that are heterogeneous in clinical manifestations that develop as a result of pathological production of autoimmune antibodies or the multiplication of autoaggressive clones of killer cells against healthy, normal body tissues, leading to damage and destruction of normal tissues and the development of autoimmune inflammation. The number of autoimmune diseases ranges from 80 to 1000 according to various authors and accounts for 5\% of the world's population. Almost 400 million people are sick with diabetes mellitus alone on our planet.

The main characters of adaptive immunity - B and T - lymphocytes. They originate from bone marrow stem cells. B cells are specialized to fight bacteria, viruses, and cancer cells. They secrete proteins - antibodies that attach to disease-causing organisms and help break them down. The leading role in the autoimmune process is played by autoimmune lymphocytes, which mistake the cells of their own body for foreign ones and attack them $(18,19)$. In a healthy state, with their completely normal functioning, autoimmune lymphocytes are contained in small numbers and do not attack any structures.

Thus, in autoimmune diseases, the immune system perceives tissues as foreign elements and begins to damage them. In contrast, in the normal state of the body, the immune system exerts tight control over such a cell. But under certain conditions, for example, with aging of lymphocytes, their degeneration, degeneration of the lymphatic system as a whole, violation of their own functions in the human body, control over such cells can be lost and, as a result, they begin to act in such a way that they destroy already normal, full-fledged cells. This is how an autoimmune disease develops.

Is it possible to cure the degradation, "aging" of lymphocytes, return them to a normal, non-degenerated state and thereby exclude the possibility of lymphocytes attacking normal tissues? Lymphocytes are part of the whole lymphatic system, including the lymph nodes. Degeneration of lymph cells is part of the degeneration of the entire lymphatic system. This is why it is extremely important to heal the degenerated lymphatic system.

\section{Treating autoimmune diseases by the method of resonance of creation}

Our task was to normalize the functional state of the lymph nodes and lymphocytes in our patients and thus take an important step towards curing their autoimmune disease. To this end, we need to raise the potency of the lymph nodes and lymphocytes in the same way as we did in relation to the substantia nigra of the midbrain in patients with Parkinson's disease.

\section{Diagnosis of autoimmune diseases by the method of resonance of creation}

When testing an organ in a patient in a degenerative process, it stops being tested as normal. In other words, it is tested as problematic. At the same time, an arrow falls on the computer screen in the middle of the screen, which indicates that the organopreparation was found correctly. There is a resonance between the organopreparation and the patient. This is a diagnostic resonance, but not a therapeutic one.

\section{Creation Resonance Treatment}


The doctor finds that potency of the organopreparation in the patient, which leads to resonance with the affected organ, namely, the termination of testing this organ or organ section as problematic. In this case, the arrow stops falling on the computer screen. This is a therapeutic resonance, but not diagnostic. The doctor prepares preparations of healthy organs in the F potency for the patient, writes them on the sugar crumbs, which the patient takes. Already on the first day of treatment of all patients, it was noted that when testing their organopreparations "lymph nodes" and "lymphocytes" there was a significant noticeable shift in the normalization of their potency. From the very first days of treatment, testing the autoimmune nosode in patients showed that they were tested less and less until they stopped being tested.

The above shows that if in autoimmune diseases the lymph nodes and lymphocytes were in a state of degeneration and thereby led to the onset of the disease, their treatment contributed to the normalization of the state of the lymph nodes and lymphocytes, which opened the way for the cure of autoimmune diseases.

It is important to pay attention to the fact that it was not the destruction of degraded, aged lymphocytes that took place, but their transformation into healthy, normal cells. At the same time, the number of lymphocytes in the body did not decrease, but remained exactly the same as before their transformation.

The following section provides an illustration of the effective treatment of autoimmune diseases using the example of diabetes mellitus.

\section{Treatment of diabetes mellitus using bioresonance therapy}

\section{Reconstruction of degenerated beta cells pancreas and its lymphoid system}

In type 1 diabetes mellitus, there is a process of degeneration of the pancreas (its tail), degeneration of the lymphatic system of the pancreas and, as a consequence, an autoimmune process that leads to the death of beta cells in the tail of the pancreas $(20,21)$. In order to restore the structure and function of the pancreas, organopreparations "pancreatic tail" in the F potency, lymph nodes of the pancreas and lymphacytes are used.

It is clear that without the restoration of the beta cells of the pancreas, which produce insulin, hormone replacement therapy is not able to cure diabetes mellitus.

\section{Resonance diagnostics in diabetes mellitus type 1}

Resonance diagnostics in type 1 diabetes mellitus is fundamentally no different from resonant diagnostics of other degenerative diseases. In a patient with type 1 diabetes mellitus, organ products are tested from the tail of the pancreas, which contains beta cells, lymphocytes and lymph nodes. those. the arrow on the computer goes down in the middle of the screen (8-13). This is a diagnostic resonance, but not a therapeutic one.

\section{Treatment of type 1 diabetes mellitus by the method resonance of creation}

In the patient, the potency of the organopreparations of the tail of the pancreas, lymphocytes and lymph nodes is selected, at which the computer arrow during testing stops going down in the middle of the screen. From these potentiated organopreparations, information is recorded on sugar crumbs, which becomes a medicine for patients. As a result of the course of treatment, organopreparations "pancreatic tail", lymphocytes and lymph nodes are tested completely normally, ie. when testing them, the arrow on the computer does not go down. In patients during treatment, the blood sugar level normalizes, and therefore there is no need for insulin replacement therapy. There are no side effects or complications during treatment (8-13).

\section{Treatment of type 2 diabetes mellitus by the method of resonance of creation and resonance of destruction}

In the case of the second type of diabetes mellitus, in addition to the nosode "diabetes mellitus of the second type", those organopreparations were tested that were also detected in the first type of diabetes mellitus degenerated tail of the pancreas, lymph nodes, lymphocytes. When treating type 2 diabetes mellitus, it is necessary to treat amyloidosis. This is due to the fact that amyloid cords in this form of diabetes are located on the beta cells of the pancreas. Treatment of amyloidosis is carried out by the method of resonance destruction. As a result of the treatment of type 2 diabetes mellitus, there is a restoration of organ products of the tail of the pancreas, lymph nodes, lymphocytes and normalization of testing of the nosode "amyloidosis". Patients have normal blood sugar levels and no longer need to take other medications (8-13). 


\section{Retinal degeneration. Effective Creation Resonance Treatment}

The retina is the inner lining of the eye, which is the peripheral part of the visual analyzer, contains photoreceptor cells that provide the perception and conversion of electromagnetic radiation from the visible part of the spectrum into nerve impulses, and also provides their primary processing. Anatomically, the retina is a thin shell, adjoining throughout its entire length from the inside to the vitreous body, and from the outside to the choroid of the eyeball. In it, two parts of unequal size are distinguished: the visual part - the largest, extending to the ciliary body itself, and the anterior - not containing photosensitive cells - the blind part, in which the ciliary and iris parts of the retina are isolated, respectively, to the parts of the choroid.

The visual part of the retina has an unequal layered structure, accessible for study only at the microscopic level, and consists of 10 layers of the eyeball. Thus, the outer nuclear layer of the retina contains the bodies of photosensory cells, the inner nuclear layer contains the bodies of bipolar, horizontal and amacrine cells, and the ganglionic layer contains ganglion cells. The retina is an extremely complex structure that is connected with the visual part of the brain with the help of nerve fibers. Therefore, we can assume that the retina is part of the brain.

The most common diseases in the elderly and senile age are retinal degeneration and its extreme manifestation - retinal detachment. Retinal detachment is the separation of rods and cones. They are called neuroepithelium. So, the neuroepithelium is separated from the underlying pigment epithelium. In this case, there is an accumulation of liquid between them. With retinal detachment, the nutrition of the outer layers of the retina is disturbed, which leads to loss of vision. Currently, the diagnosis of violations of the structure of the retina is carried out by Optical Coherence Tomography (OCT). So, retinal degeneration can be with and without retinal detachment $(22,23)$.

Patient F, 73 years old, during examination by an ophthalmologist, revealed retinal degeneration without detachment. Optical coherence tomography of the retina confirmed and documented the process of degeneration of the retina in its initial form (see photos $1 \mathrm{~A}$ and $\mathrm{B}$.

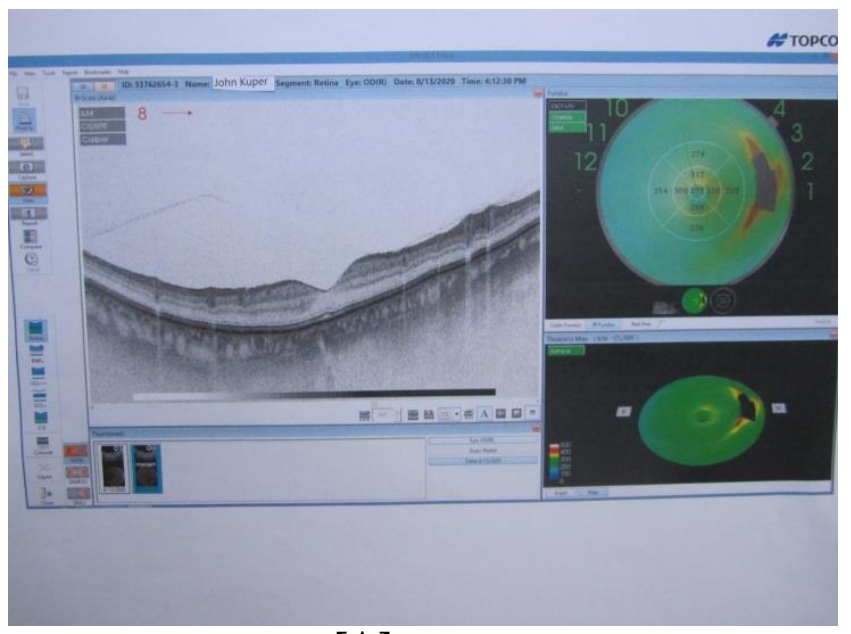

[A]

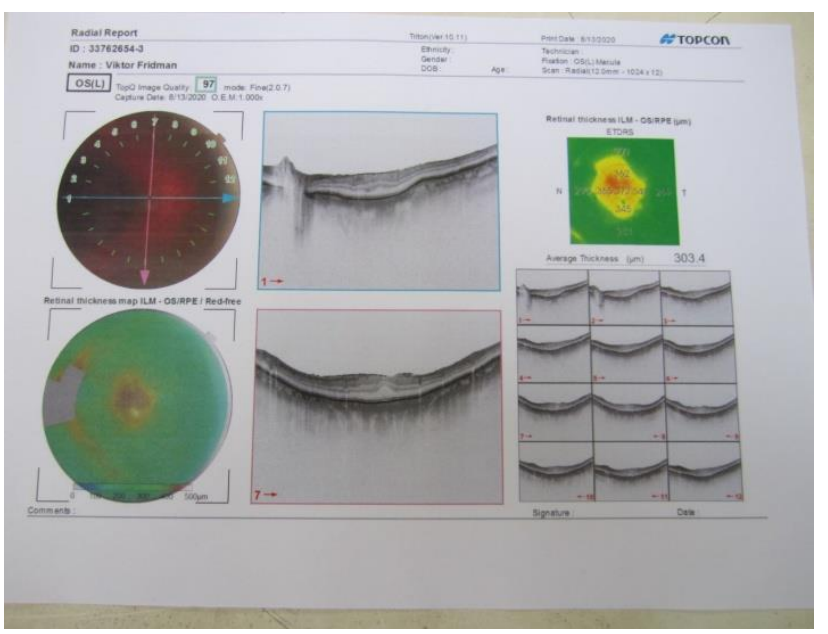

[B]

Photo 1. A and B. Optical coherence tomography of the retina of the patient F-n 73 years old before the treatment with the method of bioresonance therapy.

In addition, the examination showed a significant deterioration in visual acuity in the eye where the retinal degeneration occurred.

With retinal dystrophy, there is a thickening of its neurosensory part due to multiple small "cylindrical" cavities with hyporeflective contents, the absence of vitreoretinal fusion. On optical coherence tomography of the retina, with its degeneration, a fibrovascular membrane of a low degree of activity appears which can be seen in the photo. No. 1. In addition, there is 1 . a decrease in the thickness of the neuroepithelium in the fovea, 2 a decrease in the macular volume, 3. a pronounced decrease in the reflectivity of the neuroepithelium in the fovea. 


\section{Praznikov Viktor / Resonant Medicine}

In addition to complaints of visual impairment, the patient noted that he experiences sand in the eye, mucus from the nose (morning runny nose) in the morning, constant noise in the left ear for six years, recurrent itching in the left auricle. The patient also suffers from Parkinson's disease.

\section{Treatment of retinal degenerative changes. Restoration of the retina using the resonance of creation}

The retina was tested on a device for bioresonance therapy and it was found that the organopreparation "retina" is being tested in our patient, which indicates that there is at least degenerative changes in the retina - the arrow on the computer screen falls in the middle of the screen. The optic nerve was tested in patient Fn 73 years old and in all our other patients. A completely distinct testing of the optic nerve and the visual cortex of the brain was found, which indicates that with this disease, degeneration of the optic nerve and visual cortex of the brain takes place, which requires their restoration.

The treatment plan included the selection of the potency of the organopreparations of the "retina", the optic nerve and the visual cortex of the brain. It is clear that the changes occurring in the retina are associated with the fact that antibodies to the retina circulate in the blood and lacrimal fluid, which lead to even greater degeneration. If these antibodies are not eliminated, then pathological changes in the retina will be chronic. To this end:

[1] The potency of the organopreparation "retina" was selected, at which the drug stopped being tested. The preparation was made, which was taken by the patient. As a result of the treatment, the organopreparation "retina" was no longer tested; she, the retina, has recovered.

[2] Carried out the selection of the potency of the organopreparation "optic nerve". A preparation was made, which the patient was taking, as a result of which the organ preparation "optic nerve" was no longer tested.

[3] As a result of the selection of the potency of the organopreparation "cerebral cortex", the manufacture of the drug and the treatment of the patient with this drug, the drug was no longer tested, which testified to the effective treatment of the cerebral cortex.

[4] The eyes do not have their own lymphatic system as well as the brain. This is why the doctor used the lymph nodes of the neck and the lymph nodes behind the ear for diagnosis and treatment.

Autonosode preparation. We used potentiated drugs of the lymphatic system, which were tested. The potency of the drugs of the lymphatic system (lymph nodes of the neck and behind the ear) was selected, at which the degenerated drug "retina" was no longer tested. The drug was used to treat a patient. After treatment, the patient did not complain of "sand in the eyes", morning runny nose and recurrent itching in the left auricle. The tinnitus has become significantly less. The visual acuity test after the treatment showed completely normal results.

The eye doctor at the second visit during the examination did not find any changes in the retina, and Optical Coherence Tomography confirmed the absence of degenerative changes in the eye (see Fig. No. 2 A and B). A simple comparison of the tomograms of the retina before and after treatment shows significant positive changes, which indicate that after the end of treatment the retina has become completely normal (13). 


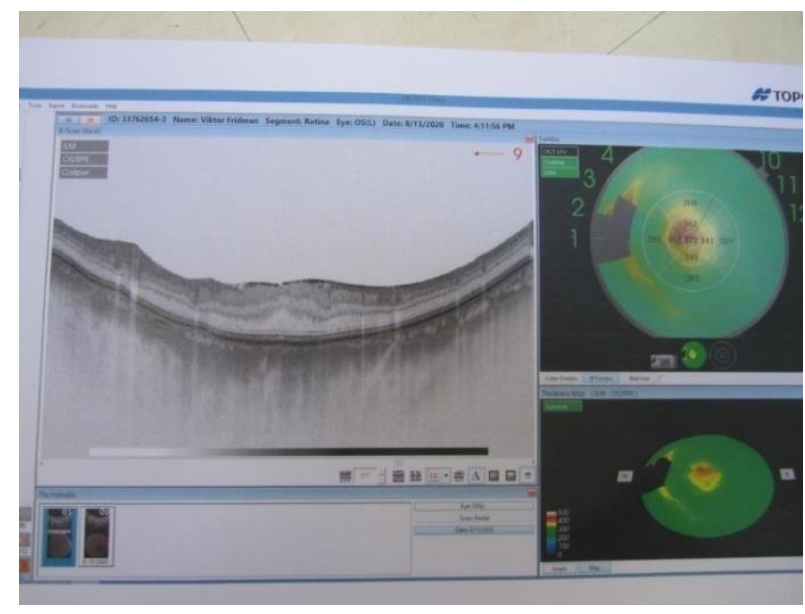

[A]

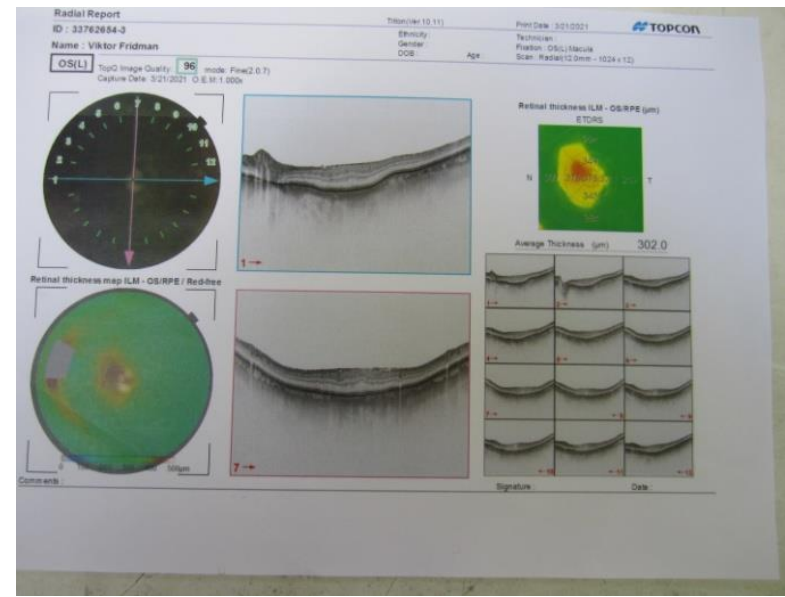

[B]

Photo 2 A and B Optical coherence tomography of the retina of the patient F-n 73 years old after treatment with the method of bioresonance therapy.

The presented tomograms are a clear illustration of treatment using the principle of "resonance of creation" (13). In addition to the aforementioned diseases, other diseases can be effectively treated with the help of the resonance of creation: Dementia, Multiple Sclerosis, Cerebral Palsy, Vitiligo, Neuritis of the auditory nerve, Amyloidosis (8-13).

With the help of the resonance of destruction, oncological diseases, prion diseases, fungal diseases, psoriasis, calculous process in the gallbladder and kidneys, cysts of the brain and kidneys are effectively treated (8-13).

\section{Conclusion:}

In this review, you can see materials about the most diverse possibilities of using resonance in medicine. The scientific substantiation of such directions as Homeopathy, resonance methods of diagnostics and treatment of various diseases is presented. It was established for the first time that not only the resonance of destruction, but also the resonance of creation is presented in nature. The possibility of using high and extremely high potencies of nosodes and organopreparations for diagnostics and treatment is shown. The high efficiency of treatment of many incurable diseases such as diabetes mellitus, Parkinson's disease, Alzheimer's disease, multiple sclerosis, serious oncological diseases, HIV infection, autoimmune diseases and many other diseases has been established.

The use of the creative resonance method made it possible to avoid the so-called substitution therapy in type 1 diabetes mellitus (insulin treatment), Parkinson's disease (dopamine treatment) and other diseases, and to use restorative therapy. The above indicates that the era of substitution therapy is ending, which is changing into the era of purely restorative treatment.

Thus, the materials of this review article meet the three principles of evidence-based medicine - scientific (resonance - scientific non-direction), efficiency and safety.

\section{Literary index:}

[1] Andrea Frova and VaripieraMarenzana. Thus spoke Galileo: the great scientist, s idea and their relevance Present day - Oxford University Press 2006. P. 133-137.

[2] Schooler, J.W., Smallwood, J., Christoff, K, Handy, T.C., Reichle, E.D., \&Sayette, M.A. (2011) Meta-awareness, perceptual decoupling and the wandering mind. Trends in Cognitive Sciences 15, 319-326.

[3] Rekweg G.-G. Homeopathic antihomotoxicology: Systematized practical medicine science. - M .: Book publishing house "Homeopathic Medicine" 2000, -592 s. (rus)

[4] Schimmel H.W. FunktionaleMedizin. V. 1,2 - Hang Verlag, Heidelberg, 1991. 


\section{Praznikov Viktor / Resonant Medicine}

[5] Louis de Brool. A revolution in physics. Atomizdat, Moscow, 1965 (rus)

[6] 15. Martinson L.K., Smirnov E.V. Section 2.2. Experimental confirmation of de Broglie's hypothesis // Quantum Physics. - M .: MGTU im. N.E.Bauman, vol. 5., 2004 (rus)

[7] Theoretical and clinical aspects of the use of bioresonance and multiresonance therapy. Moscow, 2021 (rus)

[8] V. Praznikov Homeopathy and Homeoigiya, Sputnic, 2016, 216 p.

[9] Praznikov V. Use of drugs of high (higher LM) potency in bioresonance therapy. The principle of Homeoigy and its relationship with Homeopathy in bioresonance therapy. "Sputnik +", Moscow, 2017, 220 p. (rus)

[10] Praznikov V.P. Effective treatment of cancer , degenerative and infectious diseases of the drug tami of high potencies. Moscow, "Sputnik +", 2018, 254 p. (rus)

[11] Praznikov V. Resonant medicine 1. Resonance of destruction - effective treatment of oncological, infectious diseases, cysts, etc. Resonance of creation - effective treatment of degenerative diseases - diabetes mellitus, Parkinson's disease, multiple sclerosis, etc. Moscow, "Sputnik +" 2019232 p. (rus)

[12] Praznikov V. Resonance Medicine 2. The use of resonance destruction for effective of oncological treatment, infection diseases, cysts and etc. The use of resonance creation for effective treatment of degenerative diseases - diabetes, Parkinson desease, multiple scleroses and etc. Moscow, "Sputnic +", 2020, 298 p.

[13] Praznikov V. Resonance Medicine 3. The use of resonance destruction for effective treatment of oncological, infection diseases? Cysts and etc. The use of resonance creation for effective treatment degenerative diseases - diabetes vellitus? Alzgeimer, s disease, Parkinson, s disease, multiple sclerosis, etc. Effective treatment of autoimmune diseases. "Sputnik +" 2021, 350 p.

[14] Illarioshkin S.N., Levin O.S., FedotovU.Yu., Kolokolov O.V. Parkinsonism and substantia nigra. M., "Atmosphere" 2019, 304 pp. (rus).

[15] Guidelines for the diagnosis and treatment of Parkinson's disease. Edited by Illarioshkin S.N., Levin O.S. M ,. "Patero-Print", 2017, 336 p. (rus).

[16] Gavrilova S.I. Alzheimer's disease: current understanding of diagnosis and treatment. Russian medical journal 1997, No. 20. p. 7 (rus).

[17] EislerArkady. Alzheimer's disease: diagnosis, treatment. M .: Eksmo, 2013 - 512 p. (rus)

[18] Melnikov V.L., Mitrofanova N.N., Melnikov L.V. Autoimmune diseases. Penza, PSU Publishing House, 2015230 p. (rus).

[19] Moskalev A.V., Rudoy A.S., Tsigan V.N., AlchelV.Ya. Autoimmune diseases. Diagnostics and treatment. Moscow, "GEOTAR-Media", 2020, 284 p. (rus).

[20] American Diabetes Association. Complete Giede to Diabetes 544 p.

[21] Demicheva O. Diabetes mellitus. Dr. Radionov's publishing house. M .. 345 pp. (rus).

[22] Bikbov M.M. Fayzrakhmanov R.R., Yarmukhamedova A.L. Age-related macular degeneration. M ,. April 2013196 p. (rus).

[23] Loskutov I.A. Belikova E.I., Korneeva A.V. Symptoms and syndromes in ophthalmology. M ,. "GEOTAR-Media", 2021, 244 pages (rus), 